Nephrologe 2021 · 16:261-268

https://doi.org/10.1007/s11560-021-00518-x

Angenommen: 12. Juli 2021

Online publiziert: 13. August 2021

๑) Springer Medizin Verlag GmbH, ein Teil von

Springer Nature 2021

Redaktion

Christiane Erley, Berlin

Werner Kleophas, Düsseldorf

\section{Nierenersatzverfahren bei Hochbetagten}

\author{
Ulrike Bechtel' · Mariam Abu-Tair ${ }^{2}$ \\ ${ }^{1} \mathrm{KfH}$ Kuratorium für Dialyse und Nierentransplantation e. V., Dillingen, Deutschland \\ ${ }^{2}$ Abteilung für Nephrologie und Diabetologie, Evangelisches Krankenhaus Bielefeld, Bielefeld, \\ Deutschland
}

\title{
Zusammenfassung
}

\section{In diesem Beitrag}

- Überblick

- Akutes Nierenversagen - Dialyse Hochbetagter auf der Intensivstation

- Terminales chronisches Nierenversagen Dialyse im ambulanten Bereich Der richtige Zeitpunkt

- Wahl des Nierenersatzverfahrens Apparativ oder konservativ? - Dialysemodalität

- Besonderheiten der Vorbereitung (Gefäßzugang, PD-Training)

- Behandlungsziele und Dialysedosis

- Konservative Behandlung - palliative Zielsetzung

- Therapieeinstellung mit Beendigung der Dialyse

- Prognose Hochbetagter mit und ohne Nierenersatzverfahren

Hochbetagte haben an der Hämodialyse eine 1-Jahres-Mortalität, die im Zusammenhang mit Komorbiditäten und einem Katheter als Dialysezugang 30\% übersteigt. Metaanalysen zeigen aber, dass frühzeitige Vorbereitung und individuelle Verfahrensauswahl die Morbidität und Mortalität auch im hohen Lebensalter entscheidend bessern. Mit zunehmendem Alter und Gebrechlichkeit verschieben sich dabei die Behandlungsziele weg von der Verlängerung der Lebensdauer auf die Verbesserung der Lebensqualität. Damit kann die Präferenz von Heimdialyseverfahren, auch als assistierte Peritonealdialyse, ebenso Bedeutung erringen wie die fachnephrologische Behandlung ohne Nierenersatzverfahren mit palliativem Therapieziel. Im höheren Lebensalter bestimmen zunehmend Komorbiditäten, kognitive Einschränkungen, Gebrechlichkeit und die Gesamtprognose das sinnvolle Vorgehen. Bereits bei der Anlage von Gefäßzugängen ergeben sich hinsichtlich Anastomosenort und Anlagezeitpunkt bei Hochbetagten andere Entscheidungskriterien. Empfehlungen zu Dialysedauer und -frequenz folgen der Lebensqualität mit inkrementellen und am Ende des Lebens auch dekrementellen Therapieregimen. Die demographische Entwicklung stellt die Nephrologie mit einer Zunahme älterer Patienten vor besondere Herausforderungen. Frühzeitige Aufklärung über alle Nierenersatzverfahren und die Festlegung individueller Therapieziele können bei sorgfältiger Auswahl von Dialysemodalität und -intensität auch bei Hochbetagten entscheidend zur Verbesserung der Prognose und insbesondere der Lebensqualität beitragen.

\section{Schlüsselwörter}

Dialyse $\cdot$ Alter $\cdot$ Frailty $\cdot$ Komorbidität $\cdot$ Lebensqualität

\section{Überblick}

Über 80-Jährige beginnen die Hämodialyse mit einer durchschnittlichen 2-Jahres-Mortalität von $20 \%$. Komorbiditäten wie Diabetes, kardiovaskuläre Erkrankungen, Rauchen, Übergewicht und der Dialysestart über einen Katheterzugang erhöhen bereits die 1-Jahres-Mortalität auf $25 \%$, die 2-Jahres-Mortalität sogar auf $65 \%$, und diese steigt noch weiter, wenn Anämie, erhöhtes C-reaktives Protein (CRP) und niedriges Serumalbumin dazukommen. Das belegen Daten aus dem Dialysis Outcomes and Practice Survey (DOPPS; [27]). In diese Studien sind allerdings Patienten nicht eingerechnet, die eine Transplantation oder die Peritonealdialyse (PD) als Nierenersatzverfahren gewählt und ein weit besseres Outcome haben [28]. Bei Hochbetagten hat der individuelle Grad der Gebrechlichkeit („,frailty“) außerdem einen höheren Einfluss auf die Prognose als das Alter an sich $[9,22,24]$. Es gilt daher, für die gemeinsame Entscheidung zwischen Arzt und Patient, Fachpflegekraft und Angehörigen am Ende der körpereigenen Nierenfunktion einen individuellen Weg einzuschlagen, der der letzten Lebensphase gerecht wird. 


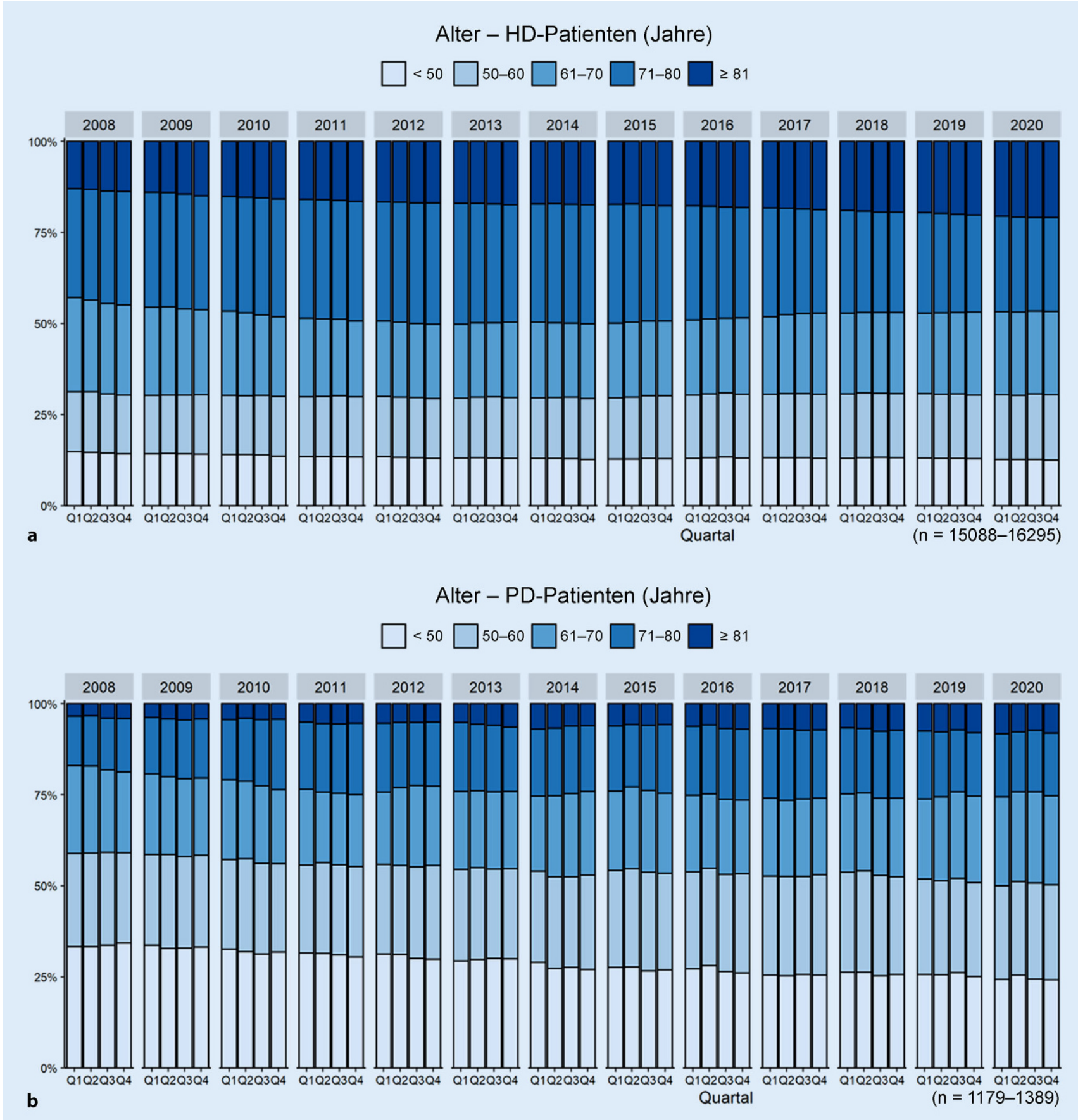

Abb. 1 ॥ Altersverteilung dialysepflichtiger Patienten im KfH Kuratorium für Dialyse und Nierentransplantation von 2008 bis 2020: a Hämo- (HD) und b Peritonealdialyse (PD). (Mit freundlicher Genehmigung, @ Matthias Schaller und Martin Wagner, QiN-Gruppe KfH und Universitätsklinik Köln)

Bei Hochbetagten unterscheiden sich viele Aspekte der Auswahl, Einleitung und Dosierung von Nierenersatzverfahren grundlegend vom Vorgehen bei jüngeren $\mathrm{Pa}$ tienten, insbesondere bei ausgedehnter Komorbidität. Die Behandlungsziele verschieben sich zunehmend weg von Verlän- gerung der Lebensdauer zur Verbesserung der Lebensqualität.

Das durchschnittliche Alter bei Dialysebeginn ist über die letzten Jahrzehnte durch die Zunahme an Sicherheit und das flächendeckende Angebot von Nierenersatzverfahren auch für Ältere und Multimorbide angestiegen. $\triangle$ Abb. 1 zeigt die
Altersverteilung Dialysepflichtiger im Verlauf von 2008 bis 2020 im KfH Kuratorium für Dialyse und Nierentransplantation e.V. in Deutschland mit einem ungebrochenen Anstieg bei den Hochbetagten ( $>80$ Jahre) sowohl in der Hämo- (HD) als auch in der Peritonealdialyse (PD). 
Hier steht eine Anzeige.

黑 Springer 
Die mit dem Alter oft einhergehende Polymorbidität und Gebrechlichkeit erhöhen oft schon vor der nephrologischen Zuweisung bei Patienten und Hausärzten die Unsicherheit, ob durch Dialyseeinleitung ein Nutzen hinsichtlich Lebensverlängerung und besonders hinsichtlich der damit zu erreichenden Lebensqualität zu erwarten ist. Für Patienten im Alter über 85 Jahre weist eine bevölkerungsbasierte Kohortenstudie aus Alberta, Kanada, eine 5-fach höhere Rate an unbehandeltem Nierenversagen nach (definiert als geschätzte glomeruläre Filtrationsrate [eGFR] $<15 \mathrm{ml} / \mathrm{min}$ ) gegenüber der Altersgruppe 18 bis 44 Jahre [17].

\section{》) Hochbetagten können Nierenersatzverfahren mit guter Lebensqualität und Prognose angeboten werden}

Die Verfasserinnen dieses Artikels möchten die vorhandenen wissenschaftlichen Grundlagen verfügbar machen für Entscheidungen, ob, zu welchem Zeitpunkt und mit welcher Modalität auch Hochbetagten ein Nierenersatzverfahren mit guter Lebensqualität und Prognose angeboten werden kann.

\section{Akutes Nierenversagen - Dialyse Hochbetagter auf der Intensivstation}

Aufgrund der demographischen Entwicklung wird der Anteil alter und sehr alter behandlungsbedürftiger Menschen deutlich steigen. Damit finden wir auch zunehmend diese Alterskohorte auf unseren Intensivstationen wieder. Komorbiditäten wie kardiovaskuläre und pulmonale Erkrankungen, aber auch eine vorbekannte chronische Niereninsuffizienz verschlechtern das Outcome dieser Patienten deutlich mit 1-Jahres-Mortalitäts-Raten von $65 \%$ bei Patienten über 90 Jahre $[3,13]$.

Grundsätzlich muss die Aufnahme auf eine Intensivstation gerade bei älteren und sehr alten Mitmenschen immer unter der Berücksichtigung von Komorbiditäten, „frailty", Prognose der Erkrankung und Schweregrad etwaiger Organdysfunktionen diskutiert werden. Auch individuelle Wünsche des Patienten müssen berücksichtigt werden.
Aufgrund der vorhandenen Logistik wird vielerorts bei notwendiger Nierenersatztherapie auf HD in Form intermittierender und kontinuierlicher Verfahren zurückgegriffen. Nichtsdestotrotz hat auch auf den Intensivstationen indikationsabhängig die PD ihren Stellenwert. Gerade aufgrund der besseren Kreislaufverträglichkeit ist sie eine sinnvolle Alternative, sofern das entsprechende Setting vorgehalten werden kann.

Patienten, die mit einem akuten Nierenversagen auf der Intensivstation mit einem Nierenersatzverfahren behandelt wurden, haben ein absolutes Risiko von $20 \%$, auch nach 1 Jahr noch dialysepflichtig zu sein [11]. Auch dieses muss bei der Prognoseabschätzung und vor Einleitung einer Nierenersatztherapie auf der Intensivstation mit in Betracht gezogen werden, setzt man sich einen größtmöglichen Erhalt an Lebensqualität als Therapieziel.

\section{Terminales chronisches Nierenversagen - Dialyse im ambulanten Bereich}

\section{Der richtige Zeitpunkt}

Aufklärungen zu allen Nierenersatzverfahren müssen in Deutschland jedem chronisch nierenkranken Patienten angeboten werden. Das Timing der Aufklärungsgespräche richtet sich nach der Geschwindigkeit des eGFR-Abfalls. Oft werden diese Gespräche in allen Altersgruppen erst bei einer eGFR von weniger als $15 \mathrm{ml} / \mathrm{min}$ initiiert, wenn die anstehende Dialysepflicht offensichtlich wird. Bei Hochbetagten ist es aus 2 Gründen sinnvoll, diese Aufklärung schon bei einer eGFR von $20-25 \mathrm{ml} / \mathrm{min}$ zu starten:

1. Für die Grundsatzentscheidung für oder gegen ein Nierenersatzverfahren braucht es oft mehrere Gespräche, u.U. auch gemeinsam mit Angehörigen. Für die Betroffenen und die Familien muss ausreichend Bedenkzeit eingeplant werden. Auch Gefäßchirurginnen und Hausärzte sollten in den Entscheidungsprozess einbezogen werden.

2. Die Auswahl der Dialysemodalität muss sorgfältig erfolgen, da der Gefäßstatus und Komorbiditäten wie Diabetes oder Herzinsuffizienz einen entscheidenden Einfluss haben. Für die Shuntreifung sind bei älteren Patienten eher 3 bis 6 Monate als 3 Wochen einzuplanen, wenn eine arteriovenöse (AV-)Fistel gelingen soll. Hochbetagte können sehr erfolgreich für ein Heimdialyseverfahren trainiert werden, wenn für zusätzliche Trainingseinheiten ausreichend Zeit zur Verfügung steht, bevor die ansteigende Urämie die Lern- und Merkfähigkeit beeinträchtigt. Eine mögliche Transplantation erfordert bei älteren Patienten oft ausgedehnte Voruntersuchungen und Sanierungen vom Zahnstatus bis zur koronaren Herzkrankheit.

\section{Wahl des Nierenersatzverfahrens}

\section{Apparativ oder konservativ?}

Vor der technischen Auswahl eines Dialyseverfahrens oder der Erwägung einer Transplantation steht bei Hochbetagten mit besonderer Intensität die Frage, ob überhaupt ein Nierenersatzverfahren eingeleitet werden soll oder eine konservative Behandlung mit palliativer Zielsetzung individuell die sinnvollere Entscheidung ist.

Die Lebenserwartung Hochbetagter ist naturgemäß geringer als die jüngerer $\mathrm{Pa}$ tienten, und die Entscheidung zur Dialyseeinleitung verlängert diese nicht immer. Es gibt gute prognostische Modelle zur Abschätzung sowohl der Kurzzeitprognose (3 bis 6 Monate) als auch der Langzeitmortalität älterer Patienten jenseits des ersten Dialysejahrs [9, 16, 27]. Möglichkeiten und Risiken der Transplantation müssen diskutiert werden.

Die Lebensqualität kann sich nach Einleitung der Dialyse zunächst verschlechtern. Stationäre Aufenthalte sind häufiger bei älteren Dialysepatienten, und die Dialyseverfahren selbst belasten Ältere oft mehr mit postinterventioneller Müdigkeit und Leistungsschwäche als Jüngere. Daher verlangt die Behandlung Hochbetagter eine besonders sorgfältige und engmaschige fachärztliche Betreuung vor und nach Einleitung der Dialyse, insbesondere in den besonders vulnerablen ersten 3 Monaten [29]. Ältere Patienten sind oft ambivalent und möchten der Dialyse erst zustimmen, wenn die einzige Alternative das unmittelbare Versterben wäre. Hier ist die frühzeitige und einfühlsame ärztliche Aufklä- 


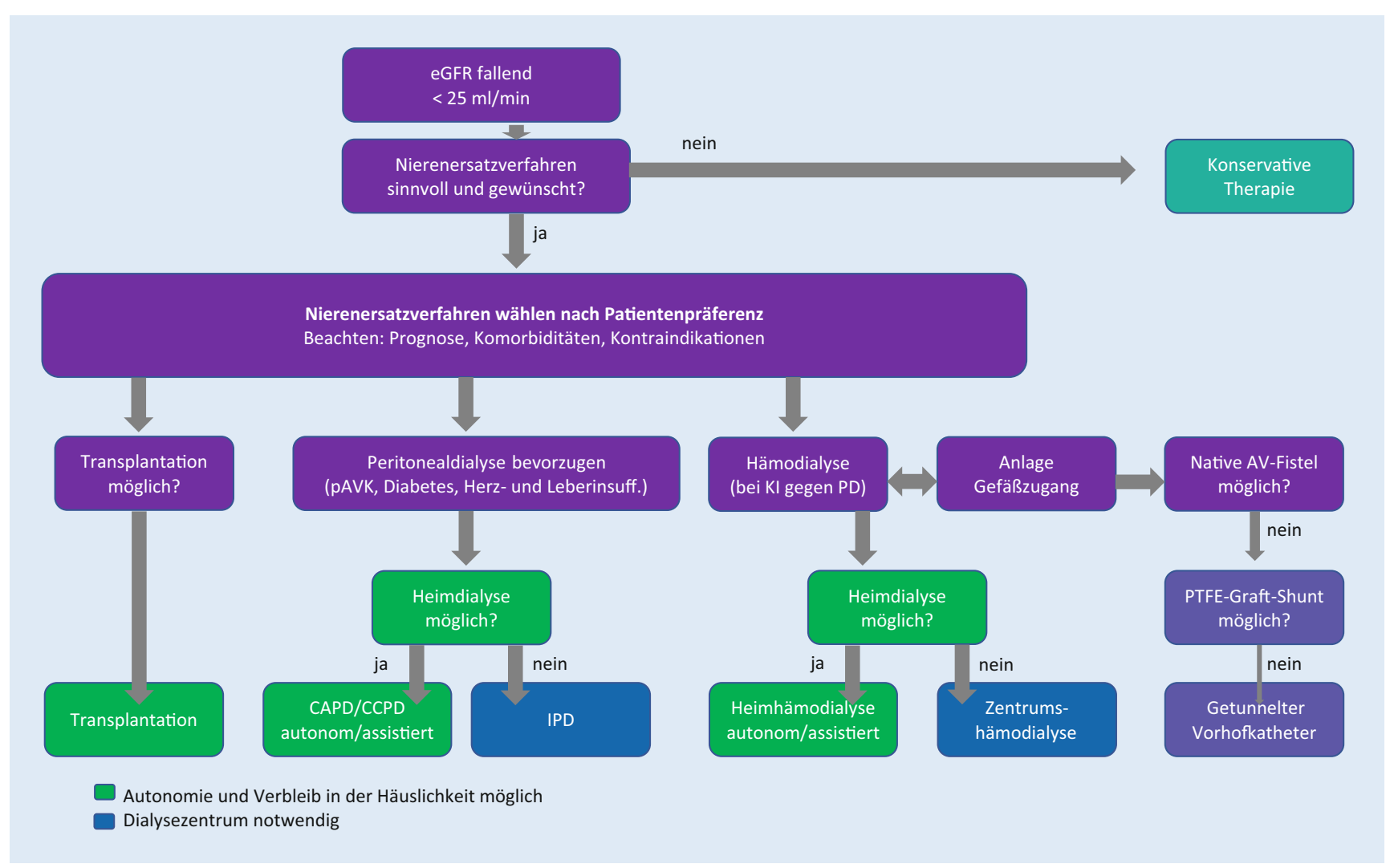

Abb. 2 \ Vorgehen zur Therapiewahl Nierenersatzverfahren bei Hochbetagten. Bei allen Verfahren inkrementelles Management erwägen; grün Autonomie und Verbleib in der Häuslichkeit möglich; blau Dialysezentrum notwendig (eGFR geschätzte glomeruläre Filtrationsrate, $p A V K$ periphere arterielle Verschlusskrankheit, KI Kontraindikation, PD Peritonealdialyse, AV-Fistel arteriovenöse Fistel, PTFE Polytetrafluorethylen, CAPD "continuous ambulatory peritoneal dialysis", CCPD "continuous cyclic peritoneal dialysis", IPD intermittierende Peritonealdialyse)

rung besonders gefordert, geht es doch mit der Vermeidung von Urämiesymptomatik und stationären Aufenthalten sowie mit der Behandlung von Anämie, Azidose und Hypervolämie auch und unmittelbar um den Erhalt der Lebensqualität.

\section{Dialysemodalität}

Bei den meisten älteren Patienten wird in Deutschland, aber auch in anderen Industriestaaten die Zentrums-HD eingeleitet, obwohl mehrere Studien belegen, dass dieses Nierenersatzverfahren Hochbetagten keinen Mortalitätsvorteil bietet [18, 25, 33]. Das Dialyseverfahren sollte auch bei Hochbetagten nach der Präferenz der Patienten ausgewählt werden. Grundsätzlich sind medizinische Indikationen und Kontraindikationen zu den Dialysemodalitäten nicht altersabhängig. Mehr noch als bei Jüngeren bestimmen aber im höheren Lebensalter die Gesamtprognose, Komorbiditäten, Gebrechlichkeit und kogni- tive Fähigkeiten die Auswahlmöglichkeiten. Auch die Eingebundenheit in familiäre oder professionelle Unterstützung spielt eine Rolle, insbesondere bei den Heimdialyseverfahren. D Abb. 2 zeigt den Weg der gemeinsamen Entscheidungsfindung („shared decision making").

\section{Entscheidungsfaktor Komorbidität} Es gibt in der Literatur Hinweise darauf, dass die PD Vorteile bietet für ältere Patienten insbesondere bei Herzinsuffizienz, pulmonaler Hypertonie, peripherer arterieller Verschlusskrankheit (pAVK) und Leberzirrhose, weil die Ultrafiltration kontinuierlich und täglich erfolgt und der negative Einfluss des Shuntvolumens auf die Herzleistung vermieden werden kann. Heimdialyseverfahren haben generell den Vorteil, dass den betagten Patientinnen der 3-mal wöchentliche Transport und das Infektionsrisiko erspart werden können, die mit einer kohortierten Behandlung in einem Dialysezentrum einhergehen. Die weltweit hohe Mortalität von älteren Zentrums-HDPatienten in der SARS-CoV-2(",severe acute respiratory syndrome coronavirus 2")-Pandemie hat den Vorteil von Heimdialyseverfahren gerade auch in infektiologischer Hinsicht besonders verdeutlicht [2].

\section{Entscheidungsfaktor Patienten- präferenz und Behandlungsziel} Sowohl PD als auch Heimhämodialyse (HHD) setzen ausreichende kognitive Fähigkeiten und Mobilität der Patienten voraus. Wenn diese eingeschränkt sind, kann die sog. assistierte Peritonealdialyse eine Alternative bieten, bei der spezialisierte Pflegedienste die häusliche Dialyse unterstützen. Die Kosteneffektivität und die Verbesserung der Lebensqualität durch assistierte PD gerade älterer und gebrechlicher Patientinnen wurden in mehreren Studien nachgewiesen $[1,4,6]$. Deshalb sind bei der Auswahl der Dialysemodalität für nichtselbständige Patienten neben der eigenen Präferenz auch die 
Möglichkeiten der Familie oder professioneller Hilfsdienste entscheidend. Wenn das Behandlungsziel auch darin besteht, die Beeinträchtigung des Tagesablaufs und der Häuslichkeit durch die Dialyse möglichst gering zu halten, bieten die PD und moderne, vereinfachte Verfahren der HHD einen Vorteil [33].

\section{Entscheidungsfaktor Outcome}

Es gibt keine validen Daten, die bei Älteren einen Vorteil von PD oder HD in Bezug auf Mortalität oder technisches Verfahrensüberleben belegen [5]. Die umfangreichen retrospektiven Studien in allen Altersgruppen ergeben ein ambivalentes Bild [18, 21, 25, 32]. Nur wenige Untersuchungen fokussieren dabei auf die Lebensqualität [21] oder korrigieren für den Grad der Gebrechlichkeit [19], der bei Hochbetagten entscheidend für die Prognose ist.

\section{Besonderheiten der Vorbereitung (Gefäßzugang, PD-Training)}

Wenn es die Gefäßverhältnisse zulassen, sollten auch bei Hochbetagten zur Hämodialyse native AV-Fisteln als Zugang entsprechend den KDIGO(Kidney Disease: Improving Global Outcomes)-Guidelines bevorzugt werden. Für die Shuntreifung sollten aber eher 3 Monate als 3 Wochen veranschlagt werden. Bei noch früherer Shuntanlage verschlechtert sich die Gefäßprognose, und Komplikationen nehmen zu [15]. Um auch bei älteren Patientinnen eine native AV-Fistel mit Erfolg anzulegen, sollten interdisziplinäre Voruntersuchungen mit Doppler-/und Duplexdarstellungen der arteriellen und venösen Armgefäße das Timing und die genaue Lokalisation der Fistel vorausplanen. Dann können die Venen der vorgesehenen Operationsseite bei Blutentnahmen frühzeitig geschont werden. Eine Analyse des United States Renal Data System (USRDS) über 115.425 Patienten zeigte auch für die 67bis 79-Jährigen ein besseres Überleben mit AV-Fistel gegenüber den AV-Prothesenshunts. Der Vorteil verlor sich aber bei den über 80-Jährigen [7]. Die primäre Offenheitsrate der AV-Fisteln sinkt mit steigendem Alter. Bei den Hochbetagten über 80 Jahre lag die primäre Offenheitsrate ohne Intervention bei $40 \%$ nach 1 Jahr und bei $12 \%$ nach 3 Jahren [26]. Diese schlech- te Prognose der Gefäßzugänge betrifft besonders die Frauen unter den Älteren, aber auch Patienten mit Diabetes oder pAVK [14, 31]. In einer Metaanalyse Hochbetagter zeigten brachiocephale Fisteln mit Anastomose in Ellenbeugenhöhe eine um 12\% bessere Offenheitsrate im ersten Jahr als distale radiocephale AV-Shunts [23]. Die primären Offenheitsraten für distale und proximale AV-Fisteln lagen bei $50 \%$ bzw. $59 \%$ und die sekundären Offenheitsraten bei $65 \%$ versus $73 \%$. Die Anlage einer proximalen AV-Fistel geht aber wegen der höheren Flussvolumina gleichzeitig mit einer höheren Herzvolumenbelastung und mit dem Risiko der Minderdurchblutung der Hand i.S. eines Steal-Phänomens einher. Gerade bei Älteren sind hier Raten von $10 \%$ versus $2 \%$ berichtet für brachiocephale/ proximale versus radiocephale/distale AVShunts.

\section{I) Peritonealdialyse ist besonders für ältere Frauen und Patienten mit Diabetes oder pAVK zu bevorzugen}

Für niereninsuffiziente Patienten mit ungünstigen Gefäßverhältnissen, aber guter Überlebensprognose sind PTFE(Polytetrafluorethylen)-Graft-Shunts den Vorhofkathetern weiterhin vorzuziehen. Als dauerhafter Dialysezugang sind getunnelte Katheter denjenigen vorbehalten, bei denen eine ausgedehnte pAVK der Armgefäße, erschöpfte Venenverhältnisse, eine kurze Lebenserwartung oder eine chronische Hypotension keine Alternative lassen. Vorhofkatheter kommen aber bei Hochbetagten auch als Brücke infrage für diejenigen, die hinsichtlich einer dauerhaften Dialysebehandlung noch ambivalent sind oder deren Prognose zu Dialysebeginn bei hoher Komorbidität und Gebrechlichkeit unklar ist. Überstehen diese Patienten das kritische erste Quartal und profitieren von der Dialyse mit Besserung der Lebensqualität und der medizinischen Rahmenbedingungen, dann kann anschließend ein Shunt mit gutem Erfolg angelegt werden. Der getunnelte Vorhofkatheter verbleibt als sicherer Zugang und Zeitbrücke bis zur endgültigen Shuntreifung.

\section{Behandlungsziele und Dialysedosis}

Der Vorteil einer höheren Dialysedosis lässt sich bei der insgesamt erhöhten Mortalität der Hochbetagten nicht mehr belegen. Hier ist daher Raum für eine individuelle Behandlung mit personalisiertem Therapieziel. Wenn die Dialyse noch bei signifikanter Nierenrestfunktion begonnen werden kann, ist anfangs oft eine reduzierte Behandlungsdosis möglich. Die PD bietet mit weniger häufigen Lösungswechseln oder nächtlicher Cycler-Therapie einen besonders günstigen inkrementellen Ansatz, insbesondere wenn Icodextrin für die UItrafiltration verwendet wird. Für ältere Patienten ist der Beginn der HD mit niedrigerer Frequenz und Dauer besser verträglich.

Qualitätsvorgaben zur Dialysedauer und -frequenz sollten bei Hochbetagten und Multimorbiden flexibel sein und inkrementelle Behandlungsregime zulassen. Studien belegen, dass ein inkrementeller Dialyseansatz die Aufnahme der vollen Dialysedosis ohne Erhöhung der Mortalität um bis zu 1 Jahr hinauszögern kann [12].

\section{Konservative Behandlung - palliative Zielsetzung}

Auch die Patienten, die ein Nierenersatzverfahren ablehnen oder sich dafür aus medizinischen Gründen nicht qualifizieren, müssen engmaschig und sorgfältig fachnephrologisch betreut werden. Diätetische Beratung zur Proteinbilanz sowie Phosphat- und Kaliumrestriktion sind essenziell. Renale Folgekrankheiten wie sekundärer Hyperparathyreoidismus, Hyperurikämie mit Gichtarthritis, metabolische Azidose und renale Anämie müssen sorgfältig evaluiert und therapiert werden. Um die renale Restfunktion so lange wie möglich zu erhalten, ist eine exakte Blutdruckeinstellung unter Blockade des Renin-Angiotensin-Aldosteron-Systems günstig, die anhand von LangzeitBlutdruck-Messungen unter Vermeidung hypotensiver Phasen normnahe Werte anstrebt. Komorbiditäten erfordern ggf. eine individuelle Anpassung der Zielwerte. Unter entsprechender Aufklärung kann gegen Ende der Nierenfunktion der $\mathrm{ACE}\left(\right.$, angiotensin-converting enzyme ${ }^{\prime \prime}$ )- 
Hemmer bzw. das Sartan abgesetzt werden, um die Nierenfunktion noch einige Monate auf Kosten einer nicht mehr vorhandenen Langzeitprognose zu konservieren. Unter Einbezug der hausärztlichen Versorgung und z. B. kardiologischer oder orthopädischer Mitbehandler sollte der Verzicht auf NSAR(nichtsteroidale Antirheumatika)-haltige Analgetika und Röntgenkontrastmittel besprochen werden.

\section{Therapieeinstellung mit Beendigung der Dialyse}

Bei Hochbetagten ergeben sich vorhersehbar auch Situationen, die suggerieren, ein bei besserem Gesundheitszustand eingeleitetes Nierenersatzverfahren angesichts akuter Komorbiditäten oder schleichender Verschlechterung des Allgemeinzustands wieder einzustellen. Hilfreich sind hier bereits bei Behandlungsbeginn im Vorfeld geführte Gespräche über gewünschte Therapielimitierungen und eine Patientenverfügung. Ein abrupter Abbruch der Dialyse kann zu heftigen Urämie- und Hypervolämiesymptomen führen mit Erbrechen, Cephalgien, Dyspnoe und Blutungen. Ein dekrementelles Vorgehen mit schrittweiser Reduktion von Dialysefrequenz und -dauer erreicht in der Regel besser die palliative Zielsetzung einer weitgehenden Leidensund Symptomfreiheit am Lebensende.

\section{Prognose Hochbetagter mit und ohne Nierenersatzverfahren}

Ältere Menschen haben an der Dialyse eine stark eingeschränkte Prognose. In einer Metaanalyse über 89 Studien und 294.921 ältere Dialysepatienten (mittleres Alter: 76 Jahre) lag das 1-Jahres-Überleben nur bei $73 \%$ und nur marginal über dem konservativ-palliativen Behandlungsansatz mit 71\% 1-Jahres-Überleben [10]. Eine andere retrospektive Studie mit über 70-Jährigen kam zu einem deutlichen Vorteil im mittleren Überleben der Dialysepatienten gegenüber der konservativen Gruppe von 3,1 versus 1,5 Jahren. Aber auch in dieser Studie ergab sich kein Unterschied mehr für die über 80-Jährigen. Unabhängige Prädiktoren für eine schlechte Prognose waren ein Alter über 85 Jahre, Malnutrition, Komorbiditäten, insbesondere kardiovaskuläre Erkrankungen, Gebrechlichkeit und fehlende autonome Lebensführung, aber auch der späte Dialysebeginn, ein Dialysestart über einen Katheterzugang und die ZentrumsHD als Verfahrenswahl [30]. Kognitive Einschränkungen sind bei Dialysepatienten assoziiert mit erhöhter Sterblichkeit [8]. Darüber hinaus hat der individuelle Grad der Gebrechlichkeit hohen Einfluss auf die Prognose des Gefäßzugangs und auf Kofaktoren wie Depression und reduzierte Lebensqualität [20].

\section{I) Die Auswahl oder Ablehnung eines Nierenersatzverfahrens sollte individuell adaptiert werden}

Die Auswahl oder Ablehnung eines Nierenersatzverfahrens sollte daher sorgfältig individuell an die Lebenssituation der Hochbetagten adaptiert werden. Eine engmaschige fachnephrologische Behandlung kann die Prognose älterer Patienten mit (prä-)terminaler Niereninsuffizienz wesentlich verbessern und dazu beitragen, die Lebensqualitiät und Autonomie der Betroffenen auch beim Versagen der eigenen Nierenfunktion bis ins hohe Alter zu erhalten.

\section{Fazit für die Praxis}

- Bei frühzeitiger und individueller fachnephrologischer Behandlung kann auch Hochbetagten ein Nierenersatzverfahren mit guter Lebensqualität und Prognose angeboten werden.

- Mit zunehmendem Alter verschieben sich die Behandlungsziele weg von der Lebensdauer hin zur Verbesserung der Lebensqualität.

- Die Aufklärung über alle Nierenersatzverfahren sollte schon bei einer geschätzten glomerulären Filtrationsrate (eGFR) von 20-25 $\mathrm{ml} / \mathrm{min}$ erfolgen, ggf. unter Einbezug von Angehörigen und Pflegediensten.

- Die Auswahl der Dialysemodalität richtet sich auch im Alter nach der Präferenz der Betroffenen. Besondere Berücksichtigung finden die Prognose, die Komorbiditäten, der Grad der Gebrechlichkeit und der kognitiven Fähigkeiten sowie der Wunsch nach Verbleib in der Häuslichkeit. Hier sind auch assistierte Heimverfahren erfolgreich.

- Empfehlungen zu Dialysedauer und -frequenz richten sich nach den individuellen Therapiezielen. Inkrementelle Therapieregime können die Aufnahme der vollen
Dialysedosis ohne Erhöhung der Mortalität um bis zu 1 Jahr hinauszögern.

- Am Lebensende werden palliative Behandlungsziele eher mit einem schrittweisen Ausschleichen der Dialysedauer und -frequenz erreicht. Abruptes Absetzen des Nierenersatzverfahrens führt häufig zu schweren Urämie- und Hypervolämiesymptomen.

- Auch konservative Therapieregime erfordern zum Erhalt der Lebensqualität eine engmaschige fachnephrologische Betreuung.

\section{Korrespondenzadresse}

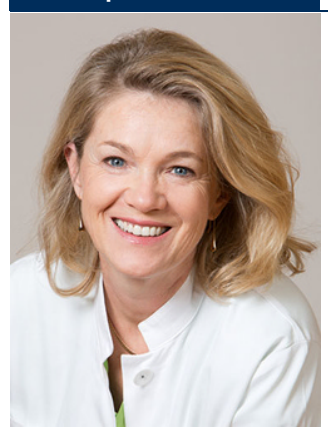

Dr. med. Ulrike Bechtel

KfH Kuratorium für Dialyse und Nierentransplantation e.V.

Ziegelstraße 38, 89407 Dillingen, Deutschland ulrike.bechtel@kfh.de

Danksagung. Die Autorinnen bedanken sich beim QiN(Qualität in der Nephrologie)-Team des KfH, Dr. Martin Wagner und Dr. Matthias Schaller, für die grafischen Abbildungen.

\section{Einhaltung ethischer Richtlinien}

Interessenkonflikt. U. Bechtel und M. Abu-Tairgeben an, dass kein Interessenkonflikt besteht.

Für diesen Beitrag wurden von den Autoren keine Studien an Menschen oder Tieren durchgeführt. Für die aufgeführten Studien gelten die jeweils dort angegebenen ethischen Richtlinien. Für Bildmaterial oder anderweitige Angaben innerhalb des Manuskripts, über die Patienten zu identifizieren sind, liegt von ihnen und/oder ihren gesetzlichen Vertretern eine schriftliche Einwilligung vor.

\section{Literatur}

1. Béchade C, Lobbedez T, Ivarsen P, Povlsen JV (2015) Assisted peritoneal dialysis for older people with end-stage-renal-disease: the French and Danish experience. Perit Dial Int 35:663

2. Bechtel U (2020) Heimdialyse - die Zukunft? Nephro News 22(4):1

3. Becker $S$ et al (2015) Clinical characteristics and outcome of very elderly patients $\geq 90$ years in 
intensive care: a retrospective observational study. Ann Intensive Care 5:53

4. Bevilaqua MU, Turnbull L, Saunders S et al (2017) Evaluation of a 12-month pilot of long-term and temporary assisted peritoneal dialysis. Perit Dial Int 37:307

5. Bieber SD, Mehrotra R (2015) Patient and technic survival of older adults with ESRD treated with peritoneal dialysis. Perit Dial Int 35:612

6. Brown EA, Dratwa M, Povlsen JV (2007) Assisted peritoneal dialysis - an evolving dialysis modality. Nephrol Dial Transplant 22:3091

7. DeSilva RN, Patibandla BK, Vin Y et al (2013) Fistula first is not always the best strategy for the elderly. J Am Soc Nephrol 24:1297

8. Drew DA, Weiner DE, Tighiouart $\mathrm{H}$ et al (2017) Cognitive decline and its risk factors in prevalent hemodialysis patients. Am J Kidney Dis 69(6):780-787

9. Floege J, Gillespie IA, Kronenberg F et al (2015) Development and validation of a predictive mortality risk score from a european hemodialysis cohort. Kidney Int 87:996

10. Foote C, Kotwal S, Gallagher M et al (2016) Survival outcomes of supportive care versus dialysis therapies for elderly patients with endstage kidney disease: a systemic review and metaanalysis. Nephrology (Carlton) 21:241

11. Forni LG, Darmon M, Ostermann M et al (2017) Renal recovery after acute kidney injury. Intensive Care Med 43(6):855-866

12. Garofalo C, Borrelli S, De Stefano T et al (2019) Incremental dialysis in ESRD: systemic review and metaanalysis. J Nephrol 32:823

13. Michels G, Sieber CC, Marx G et al (2019) Geriatrische Intensivmedizin: Konsensuspapier der DGIIN, DIVI, DGAI, DGGG, ÖGGG, ÖGIAIN, DGP, DGEM, DGD, DGNI, DGIM, DGKliPha und DGG. Z Gerontol Geriatr 52(5):440-456

14. Hinsdale JG, Lipcouritz GS, Hoover EL (1985) Vascular access in the elderly: results and perspectives in a geriatric population. Dial Transplant 14:560

15. Hod T, Patibandla BK, Vin Y et al (2015) Arteriovenous fistula placement in the elderly: When is the optimal time? J Am Soc Nephrol 26:448

16. Hapio M, Helve J, Gönhagen-Riska C, Finne P (2017) 1 - and 2-year-mortality prediction for patients starting chronic dialysis. Kidney Int Rep 2:1176

17. Hemmelgarn BR, James MT, Manns BJ et al (2012) Rates of treated and untreated kidney failure in older vs younger adults. JAMA 307:2507

18. Han SS, ParkJY, Kang Setal (2015) Dialysis modality and mortality in the elderly: a meta-analysis. Clin J Am Soc Nephrol 10:983

19. lyasere O, Brown EA (2015) Mortality in the elderly on dialysis: Is this the right debate? Clin J Am Soc Nephrol 10:920

20. Iyasere OU, Brown EA, Johansson L et al (2016) Quality of life and physical function in older patients on dialysis: a comparison of assisted peritoneal dialysis with hemodialysis. Clin J Am Soc Nephrol 11:423

21. Jeloka T, Sanwaria P, Pawer S (2016) Survival of elderly dialysis patients is not dependend on modality or" "older" age. Indian J Nephrol 26:23

22. Johansen KL, Dalrymple LS, Glidden D et al (2016) Association of performance-based and selfreported function-based definitions of frailty with mortality among patients receiving hemodialysis. Clin J Am Soc Nephrol 11:626

23. Lazarides MK, Georgiadis GS, Antoniou GA, Staramos DN (2007) A meta-analysis of dialysis

\section{Renal replacement procedures in the very old}

Very old people have a 1-year mortality on hemodialysis, which in association with comorbidities and a catheter as dialysis access exceeds 30\%; however, meta-analyses show that timely preparation and individually selected procedures decisively improve the morbidity and mortality even in old age. With increasing age and frailty the treatment targets shift away from prolongation of the lifespan to improvement of the quality of life. In this way the preference of home dialysis procedures, also as assisted peritoneal dialysis, can also achieve importance just as specialist nephrological treatment without renal replacement therapy with a palliative treatment target. In advanced age comorbidities, cognitive impairment, frailty and the overall prognosis determine the meaningful approach. Even with the placement of a vascular access there are other criteria for making decisions in very old people with respect to the anastomosis site and timing of access placement. Recommendations on the duration and frequency of dialysis follow the quality of life with incremental and at the end of life also with decremental treatment regimens. The demographic development is a special challenge for nephrology with an increase in older patients. Timely clarification of all renal replacement procedures and establishment of individual treatment targets with a careful selection of the dialysis modality and intensity can make a decisive contribution to improvement of the prognosis and particularly to the quality of life even in the very old.

\section{Keywords}

Dialysis · Age · Frailty · Comorbidity · Quality of life

access outcomes in elderly patients. J Vasc Surg 45:420

24. McAdams-DeMarco MA, Law A, Salter ML et al (2013) Frailty as a novel predictor of mortality and hospitalization in individuals of all ages undergoing hemodialysis. J Am Geriatr Soc 61:896

25. Mehrotra R, Chiu YW, Kalantar-Zadeh $\mathrm{K}$ et al (2011) Similar outcomes with hemodialysis and peritoneal dialysis in patients with end-stage renal disease. Arch Intern Med 171:110

26. Misskey J, Faulds J, Sidhu R et al (2018) An agebased comparison of fistula location, patency, and maturation for elderly renal failure patients. J Vasc Surg 67:1491

27. Ramspek CL, Voskamp PW, van Ittersum FJ et al (2017) Prediction models for the mortality risk in chronic dialysis patients: a systemic review and independent external validation study. Clin Epidemiol 9:451

28. Steenkamp R, Caskey FJ (2015) "How long have I got doctor?" The development and validation of a new prognostic model. Kidney Int 87:879

29. Thamer M, Kaufman JS, Zhang Y et al (2015) Predicting early death among elderly dialysis patients: development and validation of a risk score to assist shared decision making for dialysis initiation. Am J Kidney Dis 66:102

30. Verberne WR, Geers AB, Jellema WT et al (2016) Comparative survival among older adults with advanced kidney disease managed conservatively versus with dialysis. Clin J Am Soc Nephrol 11:633

31. Windhus DW, Jendrisak MD, Delmez JA (1992) Prosthetic fistula survival and complications in hemodialysis patients: effects of diabetes and age. Am JKidney Dis 19:448

32. Winkelmayer WV, Glynn RJ, Mittleman MA et al (2002) Comparing mortality of elderly patients on hemodialysis: a propensity score approach. J Am Soc Nephrol 13:2353

33. Wong B, Ravani P,Oliver MJ et al (2018) Comparison of patient survival between hemodialysis and peritoneal dialysis among patients eligible for both modalities. Am J Kidney Dis 71:344 